\title{
Evaluation Platform for MEMS-Actuated 3D-Printed Compliant Structures
}

\author{
$\mathrm{Xu}$ Chen \\ Department of Electrical and \\ Electronic Engineering \\ Imperial College London \\ London, United Kingdom \\ xu.chen18@imperial.ac.uk
}

\author{
Michail E. Kiziroglou \\ Department of Electrical and \\ Electronic Engineering \\ Imperial College London \\ London, United Kingdom \\ m.kiziroglou@imperial.ac.uk
}

\author{
Eric M. Yeatman \\ Department of Electrical and \\ Electronic Engineering \\ Imperial College London \\ London, United Kingdom \\ e.yeatman@imperial.ac.uk
}

\begin{abstract}
This paper presents experimental results on an evaluation platform for MEMS-actuated compliant structures. A combination of 3 dimensional (3D) flexure design, 3D printing of polymers with controlled stiffness is employed. A modular system design approach allows the interchange and combination of different actuation cantilevers, flexures and structure designs implemented as standalone test parts with minimal assembly requirements. The performance evaluation method includes synchronised electrical excitation and optical displacement measurements, allowing characterisation of motion amplification, dynamic response as well as actuating power transfer. As a demonstrator, a single lever compliant structure was designed, fabricated and tested on the platform to investigate how geometry and material stiffness affect performance. The experimental results reveal that significant improvement of amplification ratio and absolute phase lag can be achieved by selecting a flexure height and material composition suitable for a given application. This method of combined experimental evaluation and custom 3D design and printing is promising for optimising the design of compliant structures for MEMS sensors, actuators and energy transducers with amplified or translated motion capability.

Index Terms-piezoelectric, compliant structure, motion amplification, flexure, stiffness gradient.
\end{abstract}

\section{INTRODUCTION}

MEMS-actuators are microelectromechanical systems (MEMS) that transform signals or power from one energy domain to motion [1]. Such actuators have offered significant contributions to medical robotics for both industry and academic research. The application areas include positioning and manipulation of micro devices [2], [3], surgical robots [4], micro-robots [5] and so on. MEMS-actuators employ various mechanisms, including piezoelectric [6], electrostrictive [7], electromagnetic [2] and thermo-responsive [3]. Among these, piezoelectric devices have the advantages of fast response and high force output to weight ratio. Therefore, piezoelectricity is advantageous for high precision and high-frequency applications [2], [3], [8].

Conventional piezoelectric actuators are offered in stack [9], tube [10], bender [11], disk form [12] and so on. Benders, typically consisting of a bimorph structure, offer larger displacement, in the $10 \mu \mathrm{m}$ or even $10 \mathrm{~mm}$ range. Further increase of their motion range can be achieved by employing mechanical systems that amplify or translate a given motion input. Such systems are based on one or more leverage mechanisms, such as direct lever, beam buckling, or expansion mismatch between stacked material layers [13]. They can improve the performance and expand the applicability of actuators, sensors and motion power transducers. Their implementation involves flexures or joints that often require high precision assembly steps and are prone to wear or damage during operation. On the other hand, compliant structures designed for a distributed bending operation can address these challenges. A systematic design approach for such mechanisms has been proposed by Howell et al. [14].

In application specific implementations, York et al. proposed a piezoelectric-actuated mirror reflection system for medical endoscopes [15]. McClintock et al. presented a controlled robotic structure coined milliDelta for vibration cancellation [8]. Suzuki et al. proposed an origami-inspired miniature manipulator for teleoperated microsurgery [4]. Amongst these, McClintock et al. and Suzuki et al. employed multiple materials mechanical structures. This approach offers high flexibility, low risk of failure and low energy loss, but often involves complex fabrication processes. Recently emerged advanced additive fabrication technologies, such as the Objet PolyJet 3D Printers of Stratasys ${ }^{\circledR}$, enable printing of compliant structures with both rigid and flexible parts, including controlled material blending for realising monolithic stiffness gradients. Such tools, in combination with systematic design [14], are promising for the realisation of advanced, MEMS-actuated compliant motion translation systems.

In this paper, an experimental platform for testing piezoelectric beam actuated compliant structures is introduced. The platform architecture, design and fabrication are discussed in Section II. The platform is used to characterise a single flexure lever motion amplifier, discussed in Section III. Conclusions and future work are given in Section IV.

\section{The Evaluation Platform}

\section{A. Architecture}

The experimental evaluation platform consists of a power supply, a PicoScope ${ }^{\circledR} 2000$ multi-meter (Pico Technology), a laser displacement sensor (LK-G5000 CCD Laser Displacement Sensor, Keyence), and a 3D-printed baseboard (Fig.1 (a)). The baseboard accommodates one piezoelectric bender, one test part, and the displacement sensor, fixed by bolts and adjustable channels which allow the combination of different piezoelectric beams and compliant structures. Similar channels are used for positioning the sensor, to adjust the displacement measurement point (Fig.1 (b)). The power supply, consisting of a function generator (Aim-TTi TG5011A Function Generator 50MHz (Sinewave) RS232) and a high voltage amplifier (WMA-300, Falco Systems), drives the piezoelectric bender via conductive copper tapes. 


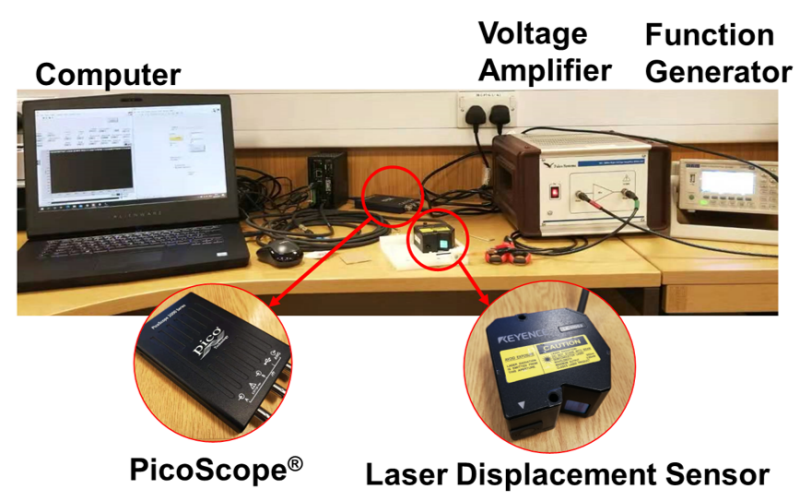

(a)



Adjustable Channels

(b)

Fig. 1. (a) Overview of the experiment platform (b) Measuring points of the laser displacement sensor. Left: Piezo-tip. Right: Compliant-tip.

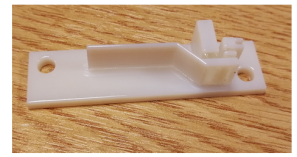

(a)

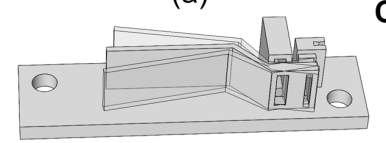

(b)

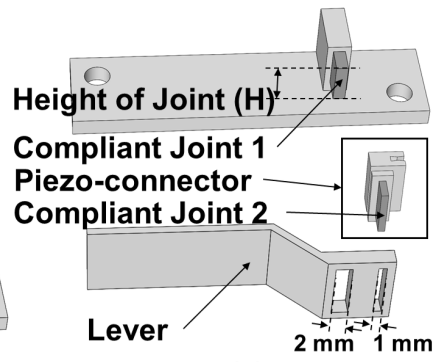

(c)
Fig. 2. (a) Photo of test pieces H795. (b) Schematic diagram of the test piece movement.(c) Names of each part and essential dimensions.

\section{B. Test Parts and Fabrication}

The test parts are 3D-printed experimental prototypes of compliant structures designed for motion translation. They include a fixing plate for assembly, and a slot for mechanical interfacing to the actuating beam. A Connex3 Objet500 MultiMaterial 3D Printer is used for rapid prototyping different designs, which allows the combination of rigid (VeroWhite ${ }^{\mathrm{TM}}$, VeroClear $^{\mathrm{TM}}$ ) and soft (Agilus30 $30^{\mathrm{TM}}$ ) polymer compounds on the same design. Furthermore, it offers controlled mixing of soft and rigid materials, realising monolithic stiffness gradients. These features provide advanced design freedom for the rapid experimental development of different types of flexures, but also for structures with distributed compliance.

TABLE I

SPECIFICATION OVERVIEW OF THE USED PIEZOELECTRIC BENDER [16]

\begin{tabular}{|l|c|l|c|}
\hline Parameters & Type 7 & Parameters & Type 7 \\
\hline $\begin{array}{l}\text { Total length } \\
{[\mathrm{mm}]}\end{array}$ & 32.5 & Total displacement [mm] & 1.4 \\
Free length [mm] & 27.5 & Blocking force [mN] & 150 \\
Width [mm] & 1.9 & $\begin{array}{l}\text { Capacity per ceramic side } \\
\text { [nF] }\end{array}$ & 13.5 \\
Thickness [mm] & 0.7 & Operating voltage [V] & 230 \\
\hline
\end{tabular}

\section{Measurement Procedure}

To measure the amplification ratio and phase lag of a given design, the displacement of the outer tip and at the piezoelectric actuator tip are recorded in two separate experiments. The signal generator waveform is set to a very low frequency, 0.1 $\mathrm{Hz}$, to minimise dynamic effects, is recorded by a PicoScope ${ }^{\circledR}$ with a LabVIEW ${ }^{\circledR}$ interface, and used for driving the phase lag between the electrical input and the output displacement. The delay introduced by the voltage amplifier was found to be negligible.

\section{Characterization of A Single Lever COMPLIANT STRUCTURE}

A flexure-based single lever mechanism was designed, fabricated and characterized on the platform using a PZT bimorph beam from Johnson Matthey (type 7). The actuator beam specifications are summarised in Table I [16]. The compliant design was based on the transmission linkage lever in [8]. Two sets of experiments were carried out to explore how the geometry and the material stiffness affect the amplification ratio and phase lag performance. The signal generator was set to $0.1 \mathrm{~Hz}, 1.5 \mathrm{~V}$ amplitude, providing 72 $\mathrm{V}$ to the beam after amplification. Designs with different heights of joint $(\mathrm{H})$ and materials were tested under the same excitation.

All test part variants have the same overall structure: a piezo-connector, a direct lever and two compliant joints with the same height but different thickness. Each piece is code-named by its joint height $(\mathrm{H})$ and the last two digits of the Connex 3 compound material code, which represent the percentage of rigid material used in the compound. For example, a design with an $\mathrm{H}$ of $7 \mathrm{~mm}$, and the Connex 3 material code FlexA4695, is called H795. The photo and CAD model of an H795 prototype are shown in Fig.2 (a) and (b). In Fig.2 (c), an exploded view is presented, showing the lever joint (Joint 1 in the Figure) and the actuation beam joint (Joint 2), which serves as a plastic interface that accommodates the surface angle change at the PZT beam. The amplified displacement at the longer side of the lever for different $\mathrm{H}$ values and soft material percentage at Joint 1 is shown in Fig. 3. The amplification ratio values of test parts are close to the theoretical expected value of 6.67 . H values were 3,5 and $7 \mathrm{~mm}$. The joint rigid material percentage was varied from $60 \%$ to $95 \%$.

\section{A. Motion Amplification Ratio}

Fig. 3 (a) and (b) present the scattered point plots of the compliant-tip amplitudes and amplification ratios of several test parts. In Fig. 3 (a), a reduction trend of displacement amplitude with increasing joint material stiffness is observed. The amplification ratio follows a similar trend for $\mathrm{H} 3$. On the contrary, H5 and $\mathrm{H} 7$ demonstrate an amplification increase with stiffness, due to a lower input displacement achieved at the lever input by the actuating beam. Effectively, the H5 and $\mathrm{H} 7$ flexures have a higher stiffness, presenting a force to the piezoelectric beam high enough to limit its motion range. The amplification ratio is higher but the overall achieved output displacement is lower than that of the $\mathrm{H} 3$ prototypes. Overall, the $\mathrm{H} 3$ prototypes provide a maximum output amplitude of $0.62 \mathrm{~mm}$, with a peak amplification ratio of 7.5 at the 70 $\%$ stiff joint material. Joints with stiffness below $70 \%$ yield reduced amplitude and amplification due to joint distortion. In 

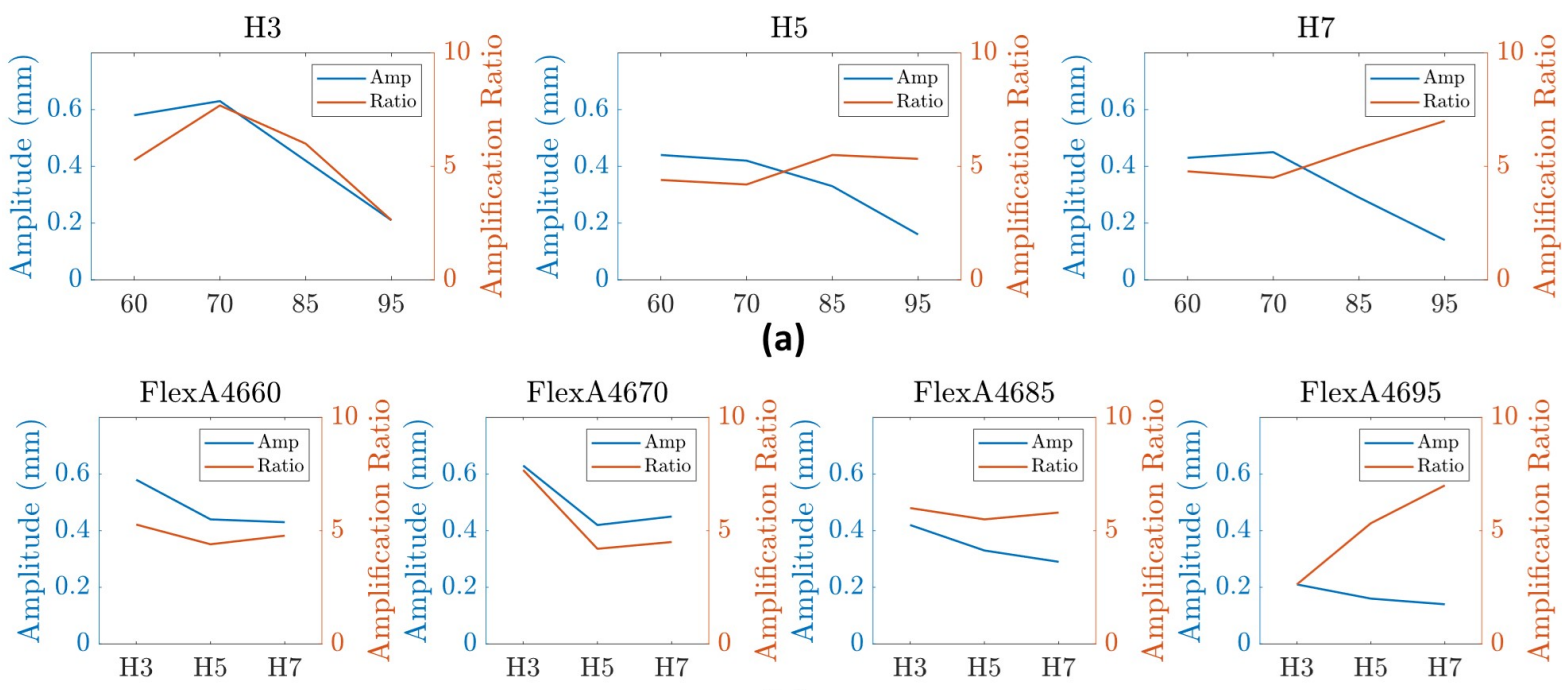

(b)

Fig. 3. Scattered point plots of Compliant-tip amplitudes and amplification ratios (a) versus the stiffness of the material; (b) versus the height of joint (H).
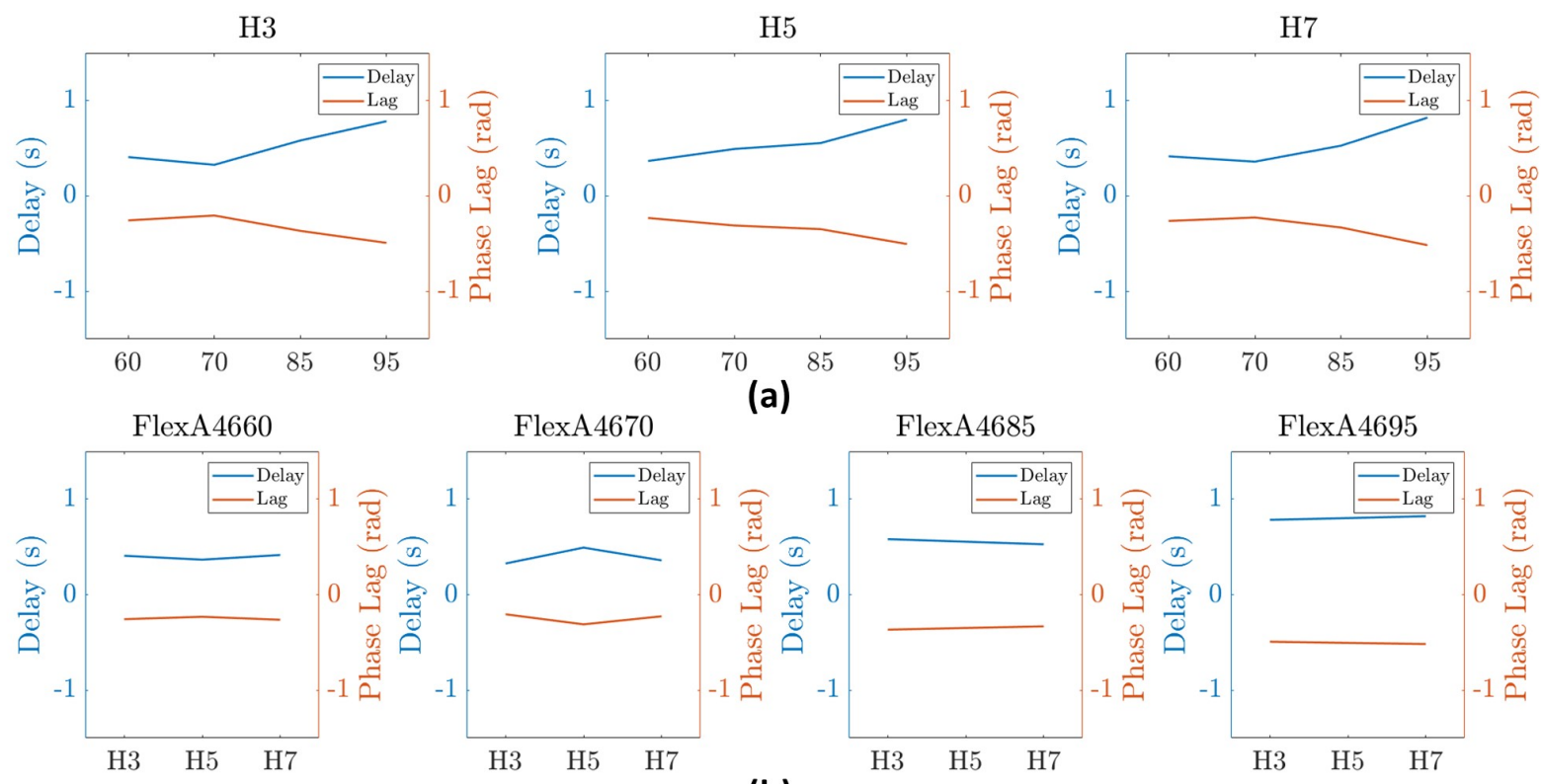

(a) FlexA4685

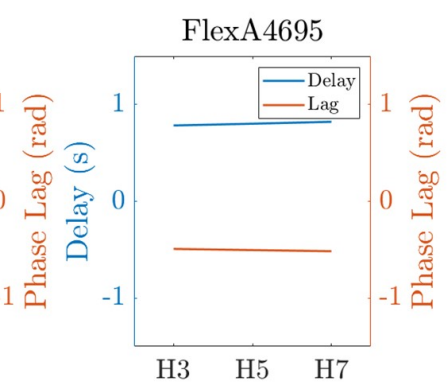

(b)

Fig. 4. Scattered point plots of time delay and phase lag of Compliant-tip displacement versus (a) the stiffness of the material; (b) the height of joint (H).

summary, force-limited (i.e. too stiff) designs show a decrease of output displacement with stiffness, whereas distortionlimited (i.e. too soft) designs exhibit an increase of output displacement with stiffness.

\section{B. Delay}

Fig. 4 (a) and (b) are scattered point plots of the time delay data and phase lag of the different designs. Time delay was calculated as the phase difference between the electrical input and the output displacement divided by the excitation angular frequency. A delay increase with material stiffness is observed. In contrast, the results do not show a significant delay dependence on joint height. The measured delay is between $0.1 \mathrm{~s}$ and $1 \mathrm{~s}(1 \%$ to $10 \%$ of the period of the applied test signal respectively) in all measurements. It is concluded that the response speed in compliant structures must be taken into account even for applications involving relatively slow (sub-
Hz) motion. The dynamic response should also be considered in the evaluation of power delivery and loss.

\section{CONCLUSION}

In this work a modular evaluation system for MEMSactuated compliant structures is introduced. The system evaluated a simple lever structure with various flexure geometries and material types. The experiments reveal that the amplification ratio of the structure varies around the theoretical value. Both amplification ratio and phase lag can be controlled by material stiffness over a wide range. In the dimension range used for the experiments, the height of joint $(\mathrm{H})$ does not affect significantly the performance of the compliant structure.

The experiments also revealed some challenges. The first is that the distortion of compliant joints dramatically affects the amplification ratio. One solution is to use a rigid (maximum stiffness), buried hinge backbone. Also, additional hinge 
structures may be required in order to minimise undesirable lateral forces and offer a smooth motion path/pattern at the interface to connect the piezo-connector and the lever. The second challenge is that the voltage amplifier phase translation needs to be taken into account in the evaluation of phase lag measurements. A circuit design with precise phase control at very low frequencies would be desirable to simplify position control, and performance evaluation of compliant structures.

For future work, compliant parallel robots would be of particular interest for biomedical and micro-robotic applications. These parallel robots can achieve several degrees of freedoms (DoFs) and controlled locking and unlocking of hinge DoFs by simple link-joint structures which can be updated by flexure-based monolithic structures. Overall, the combination of the proposed simple motion evaluation platform with MEMS actuation, flexure design and digital material 3D printing, including blended materials, could offer a new and fertile design space for motion translation devices, towards medical, robotic and power transduction micro-systems.

\section{ACKNOWLEDGMENT}

This work was financially supported by the Engineering and Physical Sciences Research Council (EPSRC), United Kingdom (EP/P012779, Micro-Robotics for Surgery). We would like to acknowledge Prof. Andrew Holmes for his measurement facilities, as well as Dr. Mohamed Abdelaziz and Mr. Jinshi Zhao for their support on LabVIEW ${ }^{\circledR}$.

\section{REFERENCES}

[1] A. S. Algamili, M. H. M. Khir, J. O. Dennis, A. Y. Ahmed, S. S. Alabsi, S. S. Ba Hashwan, and M. M. Junaid, "A Review of Actuation and Sensing Mechanisms in MEMS-Based Sensor Devices," Nanoscale Research Letters, vol. 16, no. 1, p. 16, Jan. 2021. [Online]. Available: https://doi.org/10.1186/s11671-021-03481-7

[2] Y. Huang, K. Nakamura, Y. Takida, H. Minamide, K. Hane, and Y. Kanamori, "Actively tunable $\mathrm{THz}$ filter based on an electromagnetically induced transparency analog hybridized with a MEMS metamaterial," Scientific Reports, vol. 10, p. 20807, Nov. 2020. [Online]. Available: https://www.ncbi.nlm.nih.gov/pmc/articles/ PMC7705675/

[3] O. Ulkir, "Design and fabrication of an electrothermal MEMS micro-actuator with 3D printing technology," Materials Research Express, vol. 7, no. 7, p. 075015, Jul. 2020, publisher: IOP Publishing. [Online]. Available: https://doi.org/10.1088/2053-1591/aba8e3

[4] H. Suzuki and R. J. Wood, "Origami-inspired miniature manipulator for teleoperated microsurgery," Nature Machine Intelligence, vol. 2, no. 8, pp. 437-446, Aug. 2020. [Online]. Available: http://www. nature.com/articles/s42256-020-0203-4

[5] A. Ghanbarzadeh-Dagheyan, N. Jalili, and M. T. Ahmadian, "A holistic survey on mechatronic Systems in Micro/Nano scale with challenges and applications," Journal of Micro-Bio Robotics, vol. 17, no. 1, pp. 1-22, Jun. 2021. [Online]. Available: https: //doi.org/10.1007/s12213-021-00145-8

[6] C. Liang, F. Wang, Z. Huo, B. Shi, Y. Tian, X. Zhao, and D. Zhang, "A 2-DOF Monolithic Compliant Rotation Platform Driven by Piezoelectric Actuators," IEEE Transactions on Industrial Electronics, vol. 67, no. 8, pp. 6963-6974, Aug. 2020, conference Name: IEEE Transactions on Industrial Electronics.

[7] S. John, J. Sirohi, G. Wang, and N. M. Wereley, "Comparison of Piezoelectric, Magnetostrictive, and Electrostrictive Hybrid Hydraulic Actuators," Journal of Intelligent Material Systems and Structures, vol. 18 , no. 10 , pp. 1035-1048, Oct. 2007. [Online]. Available: http://journals.sagepub.com/doi/10.1177/1045389X06072355

[8] H. McClintock, F. Z. Temel, N. Doshi, J.-s. Koh, and R. J. Wood, "The milliDelta: A high-bandwidth, high-precision, millimeter-scale Delta robot," Science Robotics, vol. 3, no. 14, Jan. 2018, publisher: Science Robotics Section: Research Article. [Online]. Available: https://robotics.sciencemag.org/content/3/14/eaar3018

[9] C. Zhou, J. Duan, G. Deng, and J. Li, "A Novel High-Speed Jet Dispenser Driven by Double Piezoelectric Stacks," IEEE Transactions on Industrial Electronics, vol. 64, no. 1, pp. 412-419, Jan. 2017, conference Name: IEEE Transactions on Industrial Electronics.
[10] S. Abdel-Gawad and X. Wang, "An improved model of thin cylindrical piezoelectric layers between isotropic elastic media," International Journal of Solids and Structures, vol. 50, no. 24, pp. 4118-4132, Nov. 2013. [Online]. Available: https://www.sciencedirect. com/science/article/pii/S0020768313003429

[11] N. Chattaraj and R. Ganguli, "Performance improvement of a piezoelectric bimorph actuator by tailoring geometry," Mechanics of Advanced Materials and Structures, vol. 25, no. 10, pp. 829-835, Jul. 2018, publisher: Taylor \& Francis eprint: https://doi.org/10.1080/15376494.2017.1308590. [Online]. Available: https://doi.org/10.1080/15376494.2017.1308590

[12] R. K. Haldkar, A. Khalatkar, V. K. Gupta, and T. Sheorey, "New piezoelectric actuator design for enhance the micropump flow," Materials Today: Proceedings, vol. 44, pp. 776-781, Jan. 2021. [Online]. Available: https://www.sciencedirect.com/science/article/pii/ S2214785320383255

[13] M. E. Kiziroglou, B. Temelkuran, E. M. Yeatman, and G.-Z. Yang, "Micro Motion Amplification-A Review," IEEE Access, vol. 8, pp. 64037-64 055, 2020, conference Name: IEEE Access.

[14] L. L. Howell, S. P. Magleby, and B. M. Olsen, Eds., Handbook of compliant mechanisms. Chichester, West Sussex, United Kingdom ; Hoboken: John Wiley \& Sons, Inc, 2013.

[15] P. A. York, R. Peña, D. Kent, and R. J. Wood, "Microrobotic laser steering for minimally invasive surgery," Science Robotics, vol. 6, no. 50, p. eabd5476, Jan. 2021. [Online]. Available: https: //robotics.sciencemag.org/lookup/doi/10.1126/scirobotics.abd5476

[16] "Bending actuators - Johnson Matthey Piezo Products GmbH." [Online]. Available: https://www.piezoproducts.com/products-solutions/ bending-actuators/ 